\title{
Effects of natural antioxidants on the stability of omega-3 fatty acids in dog food
}

\author{
Florentina Glodde $^{1}$, Mevlüt Günal ${ }^{2}$, Mary E. Kinsel ${ }^{3}$, Amer AbuGhazaleh ${ }^{1}$ \\ ${ }^{1}$ Department of Animal Science, Food and Nutrition, ${ }^{3}$ Department of Chemistry and Biochemistry, \\ Southern Illinois University. Carbondale, IL 62901, USA \\ ${ }^{2}$ Department of Animal Science, Süleyman Demirel University, 32200, Isparta, Turkey \\ aabugha@siu.edu
}

Received: January 12, $2018 \quad$ Accepted: March 9, 2018

\begin{abstract}
Introduction: The efficiency of five natural antioxidants (curcumin, cranberry, pomegranate, grape seed extract (GSE), and açai berry) in reducing lipid oxidation in dog food was compared to that of the synthetic antioxidant butylated hydroxyanisole (BHA). Material and Methods: In two different experiments content parameters were measured after 12 days of storage at $55^{\circ} \mathrm{C}$. In experiment one, the natural antioxidants were added at $0.2 \%$ and BHA at $0.02 \%$ of the food (DM basis), and samples were analysed for thiobarbituric acid-reactive substances (TBARS). In experiment two, the effects of GSE and curcumin at two admixture proportions $(0.1 \%$ and $0.2 \%$ of food DM) on omega- 3 fatty acid (FA) content were evaluated. Results: TBARS values were lower than the control $(\mathrm{P}<0.01)$ for curcumin, cranberry, pomegranate, and GSE but not for the açai berry $(\mathrm{P}>0.05)$. By day 12, although there were no significant differences $(\mathrm{P}>0.05)$ between the two curcumin treatments, they preserved higher concentrations of eicosapentaenoic acid (EPA) and docosahexaenoic acid (DHA) (P < 0.05) than the BHA and control treatments. The addition of GSE or BHA to dog food held $(\mathrm{P}<0.05)$ the concentrations of EPA higher than the control. The concentrations of EPA and DHA for the $0.2 \%$ GSE treatment were greater $(\mathrm{P}<0.05)$ than the $0.1 \%$ GSE treatment. Grape seed extract at $0.2 \%$ lost less $(\mathrm{P}<0.05)$ EPA concentration than BHA. Conclusion: The present results showed that, except for açai berry, the tested natural antioxidants could be used as a substitute for BHA in dog food.
\end{abstract}

Keywords: dog, food, omega-3 fatty acids, lipid oxidation, antioxidants.

\section{Introduction}

The pet industry is an important sector in the USA with total expenditures in 2016 of $\$ 66.75$ billion and $\$ 28.23$ billion of that spent on food (4). Dietary lipids in pet food can vary from $5 \%$ to $40 \%$ of the diet. In pet food, the fat source can be animal, vegetable, or sometimes a mixture of both. Fats associated with disorders and diseases have received more attention in recent years. In dogs, the requirements for omega- 3 and 6 fatty acids have not been documented, but may exist at certain stages in their life cycle. Numerous fatty acid supplements are often used to reduce problems with the coat and skin in dogs (5). These supplements contain a mixture of omega- 3 and 6 fatty acids, and sometimes include eicosapentaenoic acid (EPA; C20:5n3) and docosahexaenoic acid (DHA; C22:6n3) from marine sources. Processing and storage of dietary lipids result in physical and chemical changes. High levels of unsaturated fatty acids are highly susceptible to oxidation damage and rancidification causing major sensory alterations that occur during storage (7).

Oxidation of polyunsaturated fatty acids adversely affects the flavour, texture, colour, odour, and nutritional value of food during storage. It was reported that oxidised dietary lipids in growing dogs stunted growth, deteriorated antioxidant status, and weakened some immune functions (34). Synthetic antioxidants such as butylated hydroxytoluene (BHT), butylated hydroxyanisole, and ethoxyquin are often added to prevent the oxidation process. However, in recent years, the possible toxicity of synthetic antioxidants has been considered (37). Thus, natural antioxidants obtained from fruits, vegetables, spices, grains, and herbs have been of increasing interest. Plant extracts rich in polyphenols such as curcumin, cranberry, pomegranate, 
grape seed, and açai berry $(7,10,15)$ have exhibited antioxidative effects when added to food systems. No studies to our knowledge have been conducted on exploring the use of natural phenolic compounds in lipid retention of dog foods. Therefore, the main objective of this study was to evaluate the effects of curcumin, cranberry, pomegranate, GSE, and açai berry in dog food on reducing lipid oxidation.

\section{Material and Methods}

A generic dry dog food (18.0\% crude protein, $9.5 \%$ crude fat, $\max 12.0 \%$ moisture) was purchased from a local retailer and finely ground using a Thomas-Wiley laboratory mill with a $4 \mathrm{~mm}$ grinding disc. The food was then hand mixed with Virginia Prime Gold fish oil (Omega Protein Corporation, USA) and Barlean's pure flaxseed oil purchased from the local Co-op (Carbondale, USA). The oils used were natural, nonhydrogenated and unaltered containing no preservatives. The five natural antioxidants screened in this study were purchased from www.bulksupplements.com. The natural antioxidants were grape (Vitis vinifera) seed extract (GSE; > 95\% proanthocyanidins), curcumin (80\% curcumin, $15 \%-25 \%$ desmethoxycurcumin, $2.5 \%-6.5 \%$ bisdemethoxycurcumin), açai berry (Euterpe oleracea Mart.; 4:1 wet:dry açai berry extract and maltodextrin), pomegranate (Punica granatum L.; $40 \%$ ellagic acid), and cranberry (Vaccinium macrocarpon Ait; 4:1 wet:dry cranberry extract). Additionally, BHA from Sigma-Aldrich (USA) served as our positive control treatment.

Experiment one. Fish and flaxseed oils were added to the ground food at $3.1 \%$ and $1.8 \%$ of food (DM basis). The food was then divided into seven $3-\mathrm{kg}$ batches and assigned a treatment (three replicates per treatment). The control consisted of finely ground food mixed with fish and flaxseed oils with no added antioxidants. Natural antioxidants were added to the batches at $0.2 \%$ food DM and synthetic BHA was added at the legal limit of $0.02 \%$ of food DM (7). Once hand mixed, the batches were divided into $1 \mathrm{~kg}$ amounts and placed in an oven (Isotemp 200 Series Model 255D incubator, Fisher Scientific, USA) at $55^{\circ} \mathrm{C}$ in open foil steam table pans. On days 0 and 12 , a $150 \mathrm{~g}$ sample was collected from each pan into 1-gallon Zip-Loc bags, wrapped in aluminium foil, and immediately placed at $-80^{\circ} \mathrm{C}$ until analysis. The remaining $5 \mathrm{~kg}$ of food was stored at $-80^{\circ} \mathrm{C}$ for use in experiment two.

Experiment two. From the remaining $5 \mathrm{~kg}$ of food, six $500 \mathrm{~g}$ batches of the food were weighed and mixed with the same amounts of oils as outlined in experiment one. The treatments were a control (no added antioxidant), BHA at $0.02 \%$ of food DM, and either curcumin or GSE included at $0.1 \%$ and $0.2 \%$ of food DM. The food was then incubated at $55^{\circ} \mathrm{C}$ in an oven (Isotemp 200 Series model 255D incubator, Fisher Scientific, USA) for 12 days. On days 0 and 12, a $30 \mathrm{~g}$ sample from each pan was collected and placed into 1-gallon Zip-Loc bags, wrapped in aluminium foil, and then stored at $-80^{\circ} \mathrm{C}$ until fatty acid analysis. Each treatment was run in triplicate.

Thiobarbituric acid-reactive substances (TBARS) analysis. TBARS was determined by preparing samples for spectrophotometry as described by Botsoglou et al. (6). Briefly, $1 \mathrm{~g}$ of food was sampled into a $25 \mathrm{~mL}$ centrifuge tube and $8 \mathrm{~mL}$ of $5 \%$ trichloroacetic acid (TCA) was added, as well as $5 \mathrm{~mL}$ of $5 \%$ butylated hydroxytoluene (BHT) in hexane. The tubes were vortexed (Thermolyne 37600 mixer, Thermo Fisher Scientific, USA) for $30 \mathrm{sec}$ and then centrifuged (Beckman Coulter, USA) at 5,000 rpm for $3 \mathrm{~min}$. The top layer was discarded and the second layer was pipetted into a $10 \mathrm{~mL}$ screw-capped test tube, which was made up to $10 \mathrm{~mL}$ volume with $5 \%$ TCA. A $2.5 \mathrm{~mL}$ aliquot was pipetted into another screw-capped test tube to which $1.5 \mathrm{~mL}$ of $0.8 \%$ aqueous 2-thiobarbituric acid was also added. The prepared tube was incubated in a water bath for $30 \mathrm{~min}$ at $70^{\circ} \mathrm{C}$ and cooled under tap water. The samples were then run through the spectrophotometer (Fisher Genesys 5, thermo Fisher - scientific, USA) at a $535 \mathrm{~nm}$ wavelength and the absorption values recorded. Results expressed as mg malondialdehyde $/ \mathrm{kg}$ food were calculated from the standard curve of TEP (1,1,3,3-tetraethyoxypropane) standards.

Fatty acid analysis. Food samples were methylated using the $\mathrm{NaOCH}_{3}$ and $\mathrm{HCl}$ two-step procedure as outlined by Kramer et al. (20). Fatty acids were extracted by weighing out food samples to the nearest $0.75 \mathrm{mg}$ into a screw-capped, teflon-lined culture tube. The first step was adding $1 \mathrm{~mL}$ of prepared internal standard-C17:0 (heptadecaenoic acid) in benzene, followed by adding $2 \mathrm{~mL}$ of $5 \%$ sodium methoxide, $0.5 \mathrm{M}$ solution in methanol. The tubes were capped and lightly vortexed to mix and then incubated in a water bath at $50^{\circ} \mathrm{C}$ for $5 \mathrm{~min}$. After cooling for $5 \mathrm{~min}, 3 \mathrm{~mL}$ of $5 \%$ methanolic $\mathrm{HCl}$ was added and then recapped, vortexed, and incubated in a water bath at $80^{\circ} \mathrm{C}$ for another $10 \mathrm{~min}$. The tubes were cooled for $7 \mathrm{~min}$ and then $1 \mathrm{~mL}$ of hexane and $7.5 \mathrm{~mL}$ of $6 \%$ potassium carbonate was slowly added. The tubes were shaken by hand and centrifuged (DJB Labcare, England) at 1,200 rpm for $5 \mathrm{~min}$ to separate the layers. The top layer in the tubes was pipetted into $2 \mathrm{~mL}$ vials, capped, and run through a Shimadzu 20/20 gas chromatograph (Shimadzu Corporation, Japan). A famewax $30 \mathrm{~m}$ column $(0.25 \mathrm{~mm}$ ID and $0.25 \mu \mathrm{m}$; Restek, USA) was used and the injector temperature split was set at $255^{\circ} \mathrm{C}$, the column was set at $140^{\circ} \mathrm{C}$ and the flow rate specified at $30 \mathrm{~mL} / \mathrm{min}$. The temperature was programmed at $140^{\circ} \mathrm{C}$, stepped up in increments of $8^{\circ} \mathrm{C}$ until it reached $185^{\circ} \mathrm{C}$, and held for $5 \mathrm{~min}$. It was then raised in $5^{\circ} \mathrm{C}$ graduations to $240^{\circ} \mathrm{C}$ and held for $15 \mathrm{~min}$. Peaks were identified by comparing the retention times with those of the corresponding standards (Nu-Chek Prep, USA; Supelco, USA; and Larodan Fine Chemicals, Sweden).

Statistical analysis. The Statistical Analysis System (SAS Institute, Inc., USA) software package 
version 9.4 was used for statistical analysis. The data from experiment one was analysed using its proc GLM model with treatment as fixed effects and rep as a random effect. The data from experiment two was analysed using the proc GLM model with treatment and day as fixed effects and day by treatment as a random effect. Treatments means were separated by PDIFF using the $t$-test and statistically significant differences were declared at $\mathrm{P} \leq 0.05$.

\section{Results}

The effects of the six antioxidants on TBARS values are depicted in Fig. 1. After $12 \mathrm{~d}$ of storage, all supplements, except for açai berry, had significantly lower $(\mathrm{P}<0.05)$ TBARS values than the control.

The effects of curcumin on fatty acid values are presented in Table 1. Compared with the control, the concentrations of EPA and DHA were greater $(\mathrm{P}<0.05)$ in dog food incubated with curcumin. The concentrations of EPA and DHA for curcumin were also greater $(\mathrm{P}<0.05)$ compared with the BHA.

The effects of GSE on fatty acid values are presented Table 2. The concentrations of EPA was greater $(\mathrm{P}<0.05)$ with the GSE or BHA than with the control. The concentrations of EPA and DHA for the $0.2 \%$ GSE were greater $(\mathrm{P}<0.05)$ than the $0.1 \%$ GSE on day 12. Grape seed extract at $0.2 \%$ had greater $(\mathrm{P}<0.05)$ EPA concentration compared with the BHA.

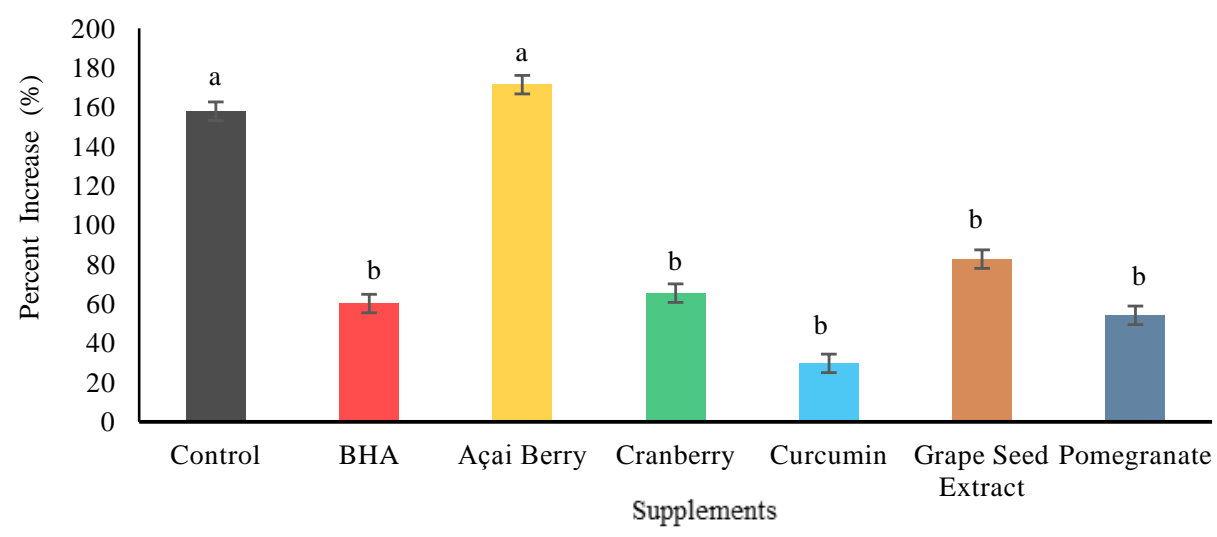

Fig. 1. The effects of BHA and natural antioxidants on the increase (\%) in TBARS for experiment one after 12 days of storage, ${ }^{\mathrm{a}-\mathrm{b}}$ Means with different letters are significantly different at $\mathrm{P}<0.05$; standard error -9.41 , BHA butylated hydroxyanisole

Table 1. Fatty acids concentrations (mg/g food DM) for BHA and the different levels of curcumin on days 0 and 12

\begin{tabular}{|c|c|c|c|c|c|c|}
\hline Fatty acid & Day & Control & BHA, $0.02 \%$ & Curcumin $0.1 \%$ & Curcumin, $0.2 \%$ & SEM \\
\hline \multirow{2}{*}{ C18:2 } & 0 & $9.67^{x}$ & $9.49^{\mathrm{x}}$ & 9.61 & 9.77 & 0.165 \\
\hline & 12 & ab $8.49^{y}$ & ${ }^{\mathrm{a}} 9.27^{\mathrm{y}}$ & ab8.74 & b8.02 & 0.174 \\
\hline \multirow{2}{*}{ C18:3n3 } & 0 & 5.99 & 6.05 & 6.09 & 5.97 & 0.097 \\
\hline & 12 & 5.73 & 5.99 & 5.76 & 5.79 & 0.889 \\
\hline \multirow{2}{*}{ C20:5n3 (EPA) } & 0 & $1.65^{x}$ & $1.71^{\mathrm{x}}$ & 1.57 & 1.58 & 0.051 \\
\hline & 12 & b $0.94^{y}$ & b1.17y & ${ }^{\mathrm{a}} 1.52$ & ${ }^{\mathrm{a}} 1.6$ & 0.056 \\
\hline \multirow{2}{*}{ C22:6n3 (DHA) } & 0 & $1.36^{\mathrm{x}}$ & $1.39^{\mathrm{x}}$ & $1.41^{\mathrm{x}}$ & $1.42^{\mathrm{x}}$ & 0.036 \\
\hline & 12 & b $0.89^{y}$ & b $1.01^{\mathrm{y}}$ & ${ }^{\mathrm{a}} 1.2^{\mathrm{y}}$ & ${ }^{\mathrm{a}} 1.08^{\mathrm{y}}$ & 0.037 \\
\hline \multirow{2}{*}{ Total omega-3 } & 0 & 8.91 & 9.08 & 9.02 & 8.91 & 0.373 \\
\hline & 12 & 7.56 & 8.23 & 8.47 & 8.32 & 0.355 \\
\hline
\end{tabular}

${ }^{\mathrm{a}-\mathrm{b}}$ Means with different subscripts within a row are significantly different at $\mathrm{P}<0.05$

${ }^{\mathrm{x}-\mathrm{y}}$ Means with different subscripts within a column are significantly different at $\mathrm{P}<0.05$

EPA - eicosapentaenoic acid; DHA - docosahexaenoic acid; BHA - butylated hydroxyanisole; SEM - standard error of the mean

Table 2. Fatty acids concentrations (mg/g food DM) for BHA and the different levels of grape seed extract (GSE) on days 0 and 12

\begin{tabular}{|c|c|c|c|c|c|c|}
\hline Fatty acid & Day & Control & BHA, $0.02 \%$ & GSE, $0.1 \%$ & GSE, $0.2 \%$ & SEM \\
\hline \multirow{2}{*}{ C18:2 } & 0 & $9.67^{x}$ & $9.49^{\mathrm{x}}$ & 9.61 & $9.77^{x}$ & 0.165 \\
\hline & 12 & b8.49y & a9.27y & ab8.9 & ${ }^{\mathrm{a}} 9.2^{\mathrm{y}}$ & 0.171 \\
\hline \multirow{2}{*}{$\mathrm{C} 18: 3 \mathrm{n} 3$} & 0 & 5.99 & 6.05 & 6.09 & 5.97 & 0.097 \\
\hline & 12 & b5.73 & ab5.99 & ${ }^{\mathrm{a}} 6.11$ & ${ }^{\mathrm{a}} 6.01$ & 0.081 \\
\hline \multirow{2}{*}{ C20:5n3 (EPA) } & 0 & $1.65^{\mathrm{x}}$ & $1.71^{\mathrm{x}}$ & $1.62^{x}$ & 1.7 & 0.046 \\
\hline & 12 & ${ }^{c} 0.94^{y}$ & $\mathrm{~b} 1.17^{\mathrm{y}}$ & $\mathrm{b} 1.23^{\mathrm{y}}$ & ${ }^{\mathrm{a}} 1.51$ & 0.048 \\
\hline \multirow{2}{*}{ C22:6n3 (DHA) } & 0 & $1.36^{\mathrm{x}}$ & $1.39^{\mathrm{x}}$ & $1.25^{\mathrm{x}}$ & 1.38 & 0.035 \\
\hline & 12 & ${ }^{\mathrm{b}} 0.89^{\mathrm{y}}$ & ${ }^{\mathrm{ab}} 1.01^{\mathrm{y}}$ & ${ }^{\mathrm{b}} 0.85^{\mathrm{y}}$ & ${ }^{\mathrm{a}} 1.23$ & 0.038 \\
\hline \multirow{2}{*}{ Total omega-3 } & 0 & $8.91^{\mathrm{x}}$ & 9.08 & 8.91 & 9.08 & 0.317 \\
\hline & 12 & b7.56 & ab 8.23 & ${ }^{\mathrm{ab}} 8.26$ & ${ }^{\mathrm{a}} 8.85$ & 0.317 \\
\hline
\end{tabular}

${ }^{\mathrm{a}-\mathrm{c}}$ Means with different subscripts within a row are significantly different at $\mathrm{P}<0.05$

${ }^{\mathrm{x}-\mathrm{y}}$ Means with different subscripts within a column are significantly different at $\mathrm{P}<0.05$

EPA - eicosapentaenoic acid; DHA - docosahexaenoic acid; BHA - butylated hydroxyanisole; GSE - grape seed extract;

SEM - standard error of the mean 


\section{Discussion}

Different analytical methods can be used for measuring lipid oxidation in foods. The thiobarbituric acid reactive substances (TBARS) method is one of the methods used to measure oxidation of fat-containing foods. Lipid oxidation can also be estimated by quantitatively measuring the loss of initial substrates. In foods containing fats or oils, unsaturated fatty acids are the main reactants whose composition changes significantly during oxidation. Changes in fatty acid composition provide an indirect measure of the extent of lipid oxidation. In a modelling study, the fatty acid concentration of various fats and oils was shown to be highly correlated with the results of four different classical assays of oxidative stability measurements (18).

In the present study, TBARS values for samples increased during storage and the increase was greater in the control samples compared with the samples treated with natural antioxidants, except for açai berry. However, previous in vitro (9) and in vivo (24) studies have reported the polyphenols of açai berry are an effective antioxidant against lipid oxidation. The antioxidant activity of extracts could be affected by factors such as storage time, extract material moisture content, temperature and time for extraction, solvent used for extraction, or method for antioxidant activity.

The present results also suggested that pomegranate is also an effective reducer of lipid oxidation as its TBARS value increased by only $54 \%$ on day 12 , compared with the $158 \%$ increase in TBARS value with the control. These results are consistent with Naveena et al. (25) who established high radical scavenging activity for both pomegranate and BHT in cooked chicken patties. Kanatt et al. (16) reported lowered TBARS values in chicken containing pomegranate extract over 20 days of storage time. Pomegranate is rich in polyphenols such as ellagitannins, gallotannins, ellagic acids, gallagic acids, catechins, anthocyanins, ferulic acids, and quercetins. The antioxidant activity of polyphenols is expressed by their redox properties that make them act as reducing agents, hydrogen donors, singlet oxygen quenchers, and also may have a metallic chelating potential. There was no statistical difference between the pomegranate and BHA treatment in our study. However, Iqbal et al. (10) observed that the addition of $0.1 \%$ pomegranate peel extract to sunflower oil reduced the TBARS value compared with this value after the addition of $0.01 \%$ BHT. Padmaja and Prasad (26) demonstrated that pomegranate extract has effective DPPH (2,2-diphenyl-1- picryl-hydrazyl) scavenging ability, higher than those of BHT and BHA.

The data obtained also confirmed the antioxidant properties of cranberry extracts. Previous studies reported reduced TBARS values in meat products containing cranberry extracts $(17,22)$. However, Sampels et al. (32) reported cranberry was not effective in inhibiting oxidation of marinating herring fillets. The present study showed no statistical differences between cranberry and BHA on TBARS formation. However, Kalin et al. (15) observed that cranberry extract inhibited lipid oxidation produced by linoleic acid emulsion to a much lower extent than BHA treatment. Cranberry contains polyphenolic compounds including phenolic acids, flavonol glycosides, anthocyanins, and proanthocyanidins. Previous studies showed that cranberry polyphenolic compounds have antioxidant activity against peroxyl (38), superoxide, hydroxyl, hydrogen peroxide, and singlet oxygen (35).

Although all of the natural antioxidants, except açai berry, tested in experiment one were effective in reducing the formation of TBARS relative to the control, only two natural antioxidants (curcumin and GSE) were selected for experiment two because of their higher availability and lower cost in comparison to the other antioxidants.

Curcumin (diferuloylmethane) is a phenolic component of Curcuma longa L. The present results suggested that the addition of curcumin to dog food reduced lipid oxidation. A study by Wang et al. (36) observed that curcumin microcapsules effectively preserved breads, tofu, and cooked pork and the authors concluded that curcumin is an effective inhibitor of oxidation and could be used as a safe and effective antioxidant. Suryanarayana et al. (33) reported that curcumin completely inhibited the formation of TBARS in the liver tissues of diabetic rats. Addition of turmeric to cooked and raw fish homogenates also reduced malonaldehyde production (8). In the present study, the lack of significant differences in the concentrations of EPA and DHA between the two curcumin treatments suggested curcumin was very effective even at the low concentration. Zhou et al. (39) reported that $40 \mathrm{ppm}$ curcumin could completely inhibit aldehyde formation in fermented cucumber tissue that was exposed to oxygen. Jayaprakasha et al. (13) observed that lipid peroxidation of linoleic acid emulsion was inhibited with $0.01 \%$ curcumin. The greater EPA and DHA concentrations for curcumin treatments compared with the BHA suggested that addition of curcumin to dog food was more effective than BHA in inhibiting lipid oxidation. A previous study by $\mathrm{Ak}$ and Gülçin (3) demonstrated that at the same concentration, curcumin was more effective than trolox, BHA and BHT in inhibiting lipid peroxidation of linoleic acid emulsion.

Commercial curcumin contains curcumin, demethoxycurcumin, and bisdemethoxycurcumin. Jayaprakasha et al. (13) reported that the antioxidant activity of the curcuminoids was curcumin $>$ demethoxycurcumin $>$ bisdemethoxycurcumin. Curcumin can act as a scavenger of free radicals (hydrogen peroxide, hydroxyl, peroxyl, peroxynitrite, singlet oxygen, superoxide, and nitrite radicals) (31). The phenolic hydroxyl group in curcumin scavenges radicals by donating its $\mathrm{H}$ atom to radicals, and the enolic hydroxyl group in curcumin can reduce radicals. Curcumin can form strong complexes with most of the known metal ions. Curcumin was also found to be a potent inhibitor of iron-induced lipid 
peroxidation (14). Previous studies have shown that curcumin and its derivatives possess antioxidant activity against DPPH radicals (29) and linoleic acid peroxidation (19).

Our results suggested that GSE is also an effective reducer of lipid oxidation. A study by Pazos et al. (28) also showed $0.01 \%$ grape polyphenols in fish lipids and frozen fish muscle was effective at lowering lipid oxidation during 26 weeks of storage. Kulkarni et al. (21) saw that at extract concentrations of $0.05 \%-0.1 \%$ GSE prevented the oxidation of beef sausage during processing and frozen storage under air. The greater EPA and DHA concentrations for the $0.2 \%$ GSE treatment compared with the $0.1 \%$ GSE treatment suggested that the antioxidant activity of GSE was dosedependent. Similarly, Ahn et al. (2) observed that the antioxidant activity of GSE was affected by the dose level. Jayaprakasha et al. (12) also found that free radical scavenging activity of the GSE was dose-dependent. In a previous study with cooked beef samples, the factor of TBARS inhibition at 6 days of storage was $36.5 \%$ for $0.02 \%$ GSE while the it was $29.5 \%$ for the samples with $0.01 \%$ added GSE (30). The greater EPA concentrations for the $0.2 \%$ GSE treatment compared with the BHA suggested that GSE could be used as a substitute for BHA in dog food. Parejo et al. (27) found that GSE exhibited greater free radical scavenging activity compared to BHA. Grape seed contains phenolic compounds such as gallic acid, ellagic acid, resveratrol, catechin, epicatechin, anthocyanins, and procyanidins (23). The antioxidative activity of GSE is mainly related to its radical scavenging and metal chelation ability, and synergism with other antioxidants (1). Antioxidative activity of GSE has been confirmed by $b$-carotene linoleate, linoleic acid peroxidation (12), DPPH, and phosphomolybdenum complex methods (11).

In conclusion, after 12 days of storage, GSE, curcumin, pomegranate, and cranberry were effective at slowing lipid oxidation. Açai berry was the only antioxidant that did not have a positive antioxidant effect on lipid oxidation. The stability of EPA and DHA in dog food also improved with the addition of GSE and curcumin. Future studies need to be aimed at finding a suitable effective dose of these supplements for inhibiting lipid oxidation in dog food.

Conflict of Interests Statement: The authors declare that there is no conflict of interests regarding the publication of this article.

Financial Disclosure Statement: This study was supported in part by an internal grant from the College of Agriculture Sciences, Southern Illinois University, USA.

Animal Rights Statement: This manuscript does not describe any studies with human or animal subjects performed by any of the authors.

\section{References}

1. Adamez J.D., Samino E.G., Sanchez E.V., Gonzalez-Gomez D.: In vitro estimation of the antibacterial activity and antioxidant capacity of aqueous extracts from grape-seeds (Vitis vinifera $\mathrm{L}$.). Food Control 2012, 24, 136-141.

2. Ahn J., Grun I.U., Fernando L.N.: Antioxidant properties of natural plant extracts containing polyphenolic compounds in cooked ground beef. J Food Sci 2002, 67, 1364-1369.

3. Ak T., Gülçin I.: Antioxidant and radical scavenging properties of curcumin. Chem Biol Interact 2008, 174, 27-37.

4. APPA. American Pet Products Association: Pet industry market size and ownership statistics. (available at: http://www.americanpetproducts.org/press_industrytrends.asp) 2017.

5. Bauer J.E.: Responses of dogs to dietary omega-3 fatty acids. J Am Vet Med Assoc 2007, 231, 1657-1661.

6. Botsoglou N.A., Fletouris D.J., Papageorgious G.E., Vassilopoulos V.N., Mantis A.J., Trakatellis A.G.: Rapid, sensitive, and specific thiobarbituric acid method for measuring lipid peroxidation in animal tissue, food, and feedstuff samples. J Agric Food Chem 1994, 42, 1931-1937.

7. Brewer M.S.: Natural antioxidants: Sources, compounds, mechanisms of action, and potential applications. Comp Rev Food Sci Food Saf 2011, 10, 1541-4337.

8. D'Souza H.P., Prabhu H.R.: In vitro inhibition of lipid peroxidation in fish by turmeric (Curcoma longa). Indian J Clin Biochem 2006, 2, 138-141.

9. Goncalves G., Santos N., Srebernich S.: Antioxidant and antimicrobial activities of propolis and açai (Euterpe oleracea Mart) extracts. Rev Ciênc Farm Básica Apl 2011, 32, 349-356.

10. Iqbal S., Haleem S., Akhtar M., Zia-ul-Haq M., Akbar J.: Efficiency of pomegranate peel extracts in stabilization of sunflower oil under accelerated conditions. Food Res Int 2008, 41, 194-200.

11. Jayaprakasha G.K., Singh R.P., Sakariah K.K.: Antioxidant activity of grape seed (Vitis vinifera) extracts on peroxidation models in vitro. Food Chem 2001, 73, 285-290.

12. Jayaprakasha G.K. Selvi T., Sakariah K.K.: Antibacterial and antioxidant activity of grape (Vitis vinifera) seed extracts. Food Res Int 2003, 36, 117-122.

13. Jayaprakasha G.K., Rao L.J., Sakariah K.K.: Antioxidant activities of curcumin, demethoxycurcumin and bisdemethoxycurcumin. Food Chem 2006, 98, 720-724.

14. Jiao Y., Wilkinson IV, J., Christine Pietsch E., Buss J.L., Wang W., Planalp R., Torti F.M., Torti S.V.: Iron chelation in the biological activity of curcumin. Free Radic Biol Med 2006, 40, 1152-1160.

15. Kalin P., Gülçin I., Gören A.C.: Antioxidant activity and polyphenol content of cranberries (Vaccinium macrocarpon). Rec Nat Prod 2015, 9, 496-502.

16. Kanatt S.R., Chander R., Sharma A.: Antioxidant and antimicrobial activity of pomegranate peel extract improves the shelf life of chicken products. Int J Food Sci Technol 2009, 45, 216-222.

17. Kathirvel P., Gong Y., Richards M.P.: Identification of the compound in a potent cranberry juice extract that inhibits lipid oxidation in comminuted muscle. Food Chem 2009, 115, 924-932.

18. Kerrihard A.L., Nagy K., Craft B.D., Beggio M., Pegg R.B.: Oxidative stability of commodity fats and oils: modeling on fatty acid composition. J Am Oil Chem Soc 2015, 92, 1153-1163.

19. Khopde S.M., Priyadarsini K.I., Venkatesan P., Rao M.N.A.: Free radical scavenging ability and antioxidant efficiency of curcumin and its substituted analogue. Biophys Chem 1999, 80, 85-91.

20. Kramer J.K., Feller V., Dugan M.E., Sauer F.D., Mossoba M.M., Yurawecz M.P.: Evaluating acid and base catalysts in the methylation of milk and rumen fatty acids with special emphasis on conjugated dienes and total trans fatty acids. Lipids 1997, 32, 1219-1228. 
21. Kulkarni S., De Santos F.A., Kattamuri S., Rossi S.J., Brewer M.S.: Effect of grape seed extract on oxidative, color and sensory stability of a pre-cooked, frozen, re-heated beef sausage model system. Meat Sci 2011, 88, 139-144.

22. Lee C.H., Krueger C.G., Reed J.D., Richards M.P.: Inhibition of hemoglobin-mediated lipid oxidation in washed fish muscle by cranberry components. Food Chem 2006, 99, 591-599.

23. Maier T., Schieber A., Kammerer D.R., Carle R.: Residues of grape (Vitis vinifera L.) seed oil production as a valuable source of phenolic antioxidants. Food Chem 2009, 112, 551-559.

24. Mertens-Talcott S.U., Rios J., Jilma-Stohlawetz P., PachecoPalencia L.A., Meibohm B., Talcott S.T., Derendorf H.: Pharmacokinetics of anthocyanins and antioxidant effects after the consumption of anthocyanin-rich acai juice and pulp (Euterpe oleracea Mart.) in human healthy volunteers. J Agric Food Chem 2008, 56, 7796-7802.

25. Naveena B.M., Sen A.R., Vaithiyanathan S., Kondaiah Y.B.N.: Comparative efficacy of pomegranate juice, pomegranate rind powder extract and BHT as antioxidants in cooked chicken patties. Meat Sci 2008, 80, 1304-1308.

26. Padmaja A., Prasad N.B.L.: Pomegranate (Punica granatum L.) peel extract as a source of natural antioxidant. J Food Eng 2011, $1,171-182$.

27. Parejo I., Viladomat F., Bastida J., Rosas-Romero A., Flerlage N., Burillo J., Codina C.: Comparison between the radical scavenging activity and antioxidant activity of six distilled and nondistilled Mediterranean herbs and aromatic plants. J Agric Food Chem 2002, 50, 6882-6890.

28. Pazos M., Gallardo J.M.M., Torres J.L., Medina I.: Activity of grape polyphenols as inhibitors of the oxidation of fish lipids and frozen fish muscle. Food Chem 2005, 92, 547-557.

29. Priyadarsini K.I., Maity D.K., Naik G.H., Kumar M.S., Unnikrishnan M.K., Satav J.G., Mohan H.: Role of phenolic OH and methylene hydrogen on the free radical reactions and antioxidant activity of curcumin. Free Radic Biol Med 2003, 35, 475-484.
30. Rojas M.C., Brewer M.S.: Effect of natural antioxidants on oxidative stability of frozen, vacuum-packaged beef and pork. J Food Quality 2007, 31, 173-188.

31. Rukkumani R., Aruna K., Varma P.S., Rajasekaran K.N., Menon V.P.M.: Comparative effects of curcumin and an analog of curcumin on alcohol and PUFA induced oxidative stress. J Pharm Sci Pharmacol 2004, 7, 274-283.

32. Sampels S., Åsli M., Vogt G., Mørkøre T.: Berry marinades enhance oxidative stability of herring fillets. J Agric Food Chem 2010, 58, 12230-12237.

33. Suryanarayana P., Satyanarayana A., Balakrishna N., Kumar P.U., Reddy G.B.: Effect of turmeric and curcumin on oxidative stress and antioxidant enzymes in streptozotocin-induced diabetic rat. Med Sci Monit 2007, 13, 286-292.

34. Turek J.J, Watkins B.A., Schoenlein I.A., Allen K.G.D., Hayek M.G., Aldrich C.G.: Oxidized lipid depresses canine growth, immune function and bone formation. J Nutr Biochem 2003, 4, 24-31.

35. Wang S.Y., Jiao H.J.: Scavenging capacity of berry crops on superoxide radicals, hydrogen peroxide, hydroxyl radicals, and singlet oxygen. J Agric Food Chem 2000, 48, 5677-5684.

36. Wang Y.F., Shao J.J., Zhou C.H., Zhang D.L., Bie Z.M., Lv F.X, Zhang C., Lu Z.X.: Food preservation effects of curcumin microcapsules. Food Control 2012, 27, 113-117.

37. Wichi H.P.: Enhanced tumor development by butylated hydroxyanisole (BHA) from the perspective of effect on forestomach and esophageal squamous epithelium. Food Chem Toxicol 1988, 26, 717-723.

38. Zheng W., Wang S.Y.: Oxygen radical absorbing capacity of phenolics in blueberries, cranberries, chokeberries, and lingonberries. J Agric Food Chem 2003, 51, 502-509.

39. Zhou A., McFeeters R.F., Fleming H.P.: Inhibition of formation of oxidative volatile components in fermented cucumbers by ascorbic acid and turmeric. J Agric Food Chem 2000, 48, 4910-4912. 